\title{
RÉPONSES GLOBALES DES INVERTÉBRÉS BENTHIQUES AUX CONDITIONS D'HABITAT PHYSIQUE DANS DES COURS D'EAU SALMONICOLES : PERSPECTIVES D'INTÉGRATION DANS DES MODĖLES HABITAT/POISSONS.
}

\author{
J.G. WASSON, R. BONNARD, L. MARIDET
}

CEMAGREF, Division Biologie des Ecosystèmes Aquatiques, Laboratoire d'Hydroécologie Quantitative, 3 bis Quai Chauveau, CP 220, 69336 Lyon Cedex 09, France.

\begin{abstract}
RÉSUMÉ
Des mesures de densité et biomasse globale provenant de 371 prélèvements répartis sur 28 sites de référence permettent d'exprimer en valeurs absolues et relatives la répartition des invertébrés en fonction des paramètres substrat, vitesse et profondeur. Les résultats diffèrent sensiblement des données existantes et permettent d'envisager la construction de courbes de préférence adaptées à une large gamme de rivières salmonicoles.
\end{abstract}

\section{GLOBAL INFLUENCE OF PHYSICAL HABITAT PARAMETERS ON BENTHIC INVERTEBRATES IN TROUT STREAMS : TOWARDS AN INTEGRATION IN FISH/HABITAT MODELS.}

\section{SUMMARY}

The influence of physical habitat parameters on macroinvertebrate densities and biomass was tested to further develop global suitability curves, in order to integrate foodproducing zones in fish/habitat models. The database contains 371 samples taken from 28 trout stream reference sites in France. Substrate diameter and structure significantly influenced densities and biomass. Values were lower on cobbles than on boulders, but sandy substrates did not differ from both. Velocity and depth significantly influenced density. Densities increased with velocity, but a minimum value occurred at $60-70 \mathrm{~cm} / \mathrm{s}$. Densities decreased with depth. Differences between observed patterns and existing curves are discussed.

\section{INTRODUCTION}

Les relations entre les macro-invertébrés benthiques et les paramètres morphodynamiques sont étudiées depuis longtemps, mais il existe peu de modèles quantitatifs reliant les invertébrés à leur habitat physique. Des courbes de préférence d'habitat ont été développés pour certaines espèces (GORE et JUDY, 1981 ; ORTH et MAUGHAN, 1983 ; MORIN et al., 1986 ; JOWETT et RICHARDSON, 1990 ; COTTARAMUSINO et al., 1991 ; JOWETT et al., 1991 ; COLLIER, 1993). Des modèles reliant les invertébrés à la distribution statistique des forces tractrices ont également été proposés (STATZNER et al., 1988). Mais la réponse globale des peuplements d'invertébrés vis-à-vis des facteurs vitesse du courant, hauteur d'eau et substrat est encore très mal documentée. WATERS (1976) a proposé des courbes de préférence globales, reprises par JOWETT (1992), pour délimiter les zones favorables à la production de nourriture pour les poissons. MILHOUS (1990) utilise également des courbes de préférence globales pour quelques groupes (biomasse de filtreurs, prédateurs, déchiqueteurs, Chironomidae) dans des modèles de microhabitat. Mais les données qui ont servi à établir ces courbes ne sont pas publiées. 
Une analyse préliminaire des données recueillies dans 19 sites de référence en France a montré une influence discriminante des facteurs vitesse, hauteur et substrat sur les densités et biomasses globales d'invertébrés. Le but du présent article est de quantifier ces relations pour ébaucher des modèles prédictifs des relations habitat / invertébrés, dans la perspective de les intégrer dans des modèles habitat / poissons.

\section{MÉTHODES}

Sites d'études - La base de données contient 371 prélèvements d'invertébrés correspondant à 65 échantillons réalisés sur 28 sites de référence exempts de pollution et d'altérations morphologiques et hydrologiques. Ces sites sont répartis sur 23 cours d'eau situés dans différentes régions écologiques différenciées par la géologie et le climat : Alpes cristallines, massifs calcaires (Jura, Vercors), Préalpes (séries sédimentaires et dépôts fluvio-glaciaires), Massif Central granitique. Les régimes hydrologiques vont du type nivo-glaciaire au pluvial atlantique ou subméditerranéen. Les altitudes s'échelonnent entre 300 et $1300 \mathrm{~m}$.

Echantillonnage - L'échantillonnage a été réalisé entre 1983 et 1993 à raison de 1 à 4 campagnes par site. Toutes les stations ont été prospectées selon le même protocole (WASSON et al., 1981) ; lors d'une campagne, un échantillon constitué de 5 ou 6 prélèvements est réalisé sur la station. Les prélèvements sont effectués au filet Surber $\left(0,1 \mathrm{~m}^{2}\right.$, maille $\left.250 \mu \mathrm{m}\right)$ dans les habitats les plus représentés sur la station en incluant les zones de bordure, et fixés au formol $4 \%$ sur le terrain. Au laboratoire, les invertébrés sont triés (tamis $630 \mu \mathrm{m}$ ), dénombrés, séchés (4 heures à $103^{\circ} \mathrm{C}$, puis dessicateur) et pesés (précision $0,1 \mathrm{mg}$ ). Le poids sec est corrigé pour chaque taxon en fonction de la perte de poids due au formol (coefficient correcteur par famille, PHILIPPE et WASSON, données non publiées).

A l'emplacement de chaque prélèvement sont mesurées la vitesse du courant (1 à 4 points de mesure, Norme ISO 748-1979-F) et la profondeur. La structure apparente du substrat est décrite visuellement à partir d'une typologie prédéfinie. Sont pris en compte dans cette étude le diamètre des éléments dominants ( $B:$ gros galets et blocs ; $G$ : galets, graviers ; $S$ : sables. Tableau I) et le caractère ouvert (poreux) ou fermé (non poreux) du substrat.

Traitement des données - L'influence des paramètres de l'habitat sur la densité et la biomasse globale a été testée à deux niveaux : en valeurs brutes sur l'ensemble des données, et en valeurs relatives au niveau des échantillons. Pour éliminer la variabilité inter-sites et saisonnière, la densité et la biomasse de chaque prélèvement ont été divisées par les valeurs moyennes de l'échantillon correspondant : ces valeurs relatives expriment exclusivement la variabilité intra-échantillon. Les moyennes, en raison de leur robustesse, ont été préférées aux maxima pour relativiser les données. Les différences entre les moyennes des variables biologiques correspondant à chaque classe de variables physiques ont été testées au seuil $p<0,05$ par des méthodes non paramétriques (KruskalWallis, Mann-Whitney).

\section{RÉSULTATS}

Les densités varient entre 130 et 46530 individus $/ \mathrm{m}^{2}$ avec une moyenne de 5824 individus $/ \mathrm{m}^{2}$, et les biomasses entre 38 et $19736 \mathrm{mg} / \mathrm{m}^{2}$ avec une moyenne de $2828 \mathrm{mg} / \mathrm{m}^{2}$.

Effet substrat - Le diamètre des éléments influence les densités et biomasses absolues et relatives. Les valeurs relevées sur les gros éléments (B) sont significativement plus élevées que sur les galets-graviers $(G)$ (Tableau I). Les sables (S) présentent apparemment des valeurs plus élévées que les substrat $G$, cette différence étant plus marquée sur les biomasses du fait d'individus plus gros dans les sables. Mais du fait d'une forte variabilité des peuplements et du petit nombre de prélèvements dans les sables, les différences entre sables et galets-graviers ne sont jamais significatives. 
Tableau I : Moyennes ( \pm erreur standard) des densités et biomasses absolues et relatives, et des biomasses individuelles des invertébrés benthiques en fonction du substrat et de la hauteur d'eau. Substrat : trois classes de diamètre (B : gros galets, blocs ; $\mathbf{G}$ : galets, graviers ; $\mathbf{S}$ : sables) et deux types de structure "ouvert" $(O)$ et "fermé" (F). Un substrat est appelé "fermé» lorsque le sable représente au moins $10 \%$ de la granulométrie apparente, ou constitue l'essentiel de la couche sous-jacente. Hauteur d'eau : six classes.

Table I : Means ( \pm standard error) of relative and absolute densities and biomass, and of the individual biomass of benthic invertebrates according to substrate and depth. Substrate : three diameter classes (B : boulders and large cobble ; $G$ : small cobble, pebble and gravel ; $S$ : sand) and two structures : «open» (ouvert) and "closed» (fermé). A substrate is labelled "closed" when sand represents more than $10 \%$ of superficial granulometry, or is largely dominant in the subsurface layer. Depth : six classes.

\begin{tabular}{|c|c|c|c|c|c|c|c|c|c|c|c|}
\hline & \multicolumn{5}{|c|}{ Types de substrat } & \multicolumn{6}{|c|}{ Classes de hauteur d'eau } \\
\hline & $\mathrm{B}$ & $G$ & $S$ & ouvert $(\mathrm{O})$ & fermé (F) & $\mathrm{H} 1$ & $\mathrm{H}_{2}$ & $\mathrm{H} 3$ & $\mathrm{H} 4$ & $\mathrm{H} 5$ & $\mathrm{H} 6$ \\
\hline Classes & $\varnothing>20 \mathrm{~cm}$ & $0.2<\theta<20 \mathrm{~cm}$ & $D<0.2 \mathrm{~cm}$ & & & {$[0-10 \mathrm{cml}$} & {$[10-20]$} & {$[20 \cdot 30[$} & {$[30-40[$} & {$[40-50]$} & {$[50-70 \mathrm{~cm}$} \\
\hline$n$ & 176 & 162 & 33 & 288 & 83 & 14 & 102 & 118 & 75 & 44 & 18 \\
\hline $\begin{array}{l}\text { Densités } \\
\text { absolues (ind./m2) }\end{array}$ & $\begin{array}{l}6648.4 \\
\pm 403.8\end{array}$ & $\begin{array}{l}4953.5 \\
\pm 301.5\end{array}$ & $\begin{array}{c}5724.9 \\
\pm 1240.8\end{array}$ & $\begin{array}{l}6179.3 \\
\pm 291.2\end{array}$ & $\begin{array}{l}4591.5 \\
\pm 545.0\end{array}$ & $\begin{array}{l}4545.0 \\
\pm 782.2\end{array}$ & $\begin{array}{l}69.36 .9 \\
\pm 582.5\end{array}$ & $\begin{array}{l}5218.8 \\
\pm 409.5\end{array}$ & $\begin{array}{l}5821.7 \\
\pm 624.3\end{array}$ & $\begin{array}{l}5957.9 \\
\pm 605.2\end{array}$ & $\begin{array}{l}4164,4 \\
\pm 753,0\end{array}$ \\
\hline $\begin{array}{l}\text { Densités } \\
\text { relatives }\end{array}$ & $\begin{array}{c}1.1 \\
\pm 0.04\end{array}$ & $\begin{array}{c}0.9 \\
\pm 0.04\end{array}$ & $\begin{array}{r}0.96 \\
\pm 0.11\end{array}$ & $\begin{array}{c}1,04 \\
\pm 0,03\end{array}$ & $\begin{array}{c}0.87 \\
\pm 0.06\end{array}$ & $\begin{array}{l}1.14 \\
\pm 0.2\end{array}$ & $\begin{array}{c}1.09 \\
\pm 0.05\end{array}$ & $\begin{array}{c}0.92 \\
\pm 0.04\end{array}$ & $\begin{array}{c}1.02 \\
\pm 0.05\end{array}$ & $\begin{array}{r}1.03 \\
\pm 0.08\end{array}$ & $\begin{array}{c}0.71 \\
\pm 0.10\end{array}$ \\
\hline $\begin{array}{l}\text { Biomasses } \\
\text { absolues (mg/m2) }\end{array}$ & $\begin{array}{l}3086.9 \\
\pm 204.4\end{array}$ & $\begin{array}{l}2407.9 \\
\pm 184.5\end{array}$ & $\begin{array}{l}3551.5 \\
\pm 899.9\end{array}$ & $\begin{array}{l}2780.4 \\
\pm 148.9\end{array}$ & $\begin{array}{l}2996.3 \\
\pm 421.6\end{array}$ & $\begin{array}{l}2278.1 \\
\pm 485.9\end{array}$ & $\begin{array}{l}2815.1 \\
\pm 292.7\end{array}$ & $\begin{array}{l}2580.2 \\
\pm 237,9\end{array}$ & $\begin{array}{l}2707.7 \\
\pm 236,3\end{array}$ & $\begin{array}{l}3587.0 \\
\pm 552.2\end{array}$ & $\begin{array}{r}3613.6 \\
\pm 1148.5\end{array}$ \\
\hline $\begin{array}{l}\text { Biomasses } \\
\text { relatives }\end{array}$ & $\begin{array}{l}1,08 \\
\pm 0.04\end{array}$ & $\begin{array}{c}0.91 \\
10.04\end{array}$ & $\begin{array}{c}1 \\
+0.16\end{array}$ & $\begin{array}{c}1,03 \\
\pm 0,03\end{array}$ & $\begin{array}{c}0.89 \\
\pm 0,08\end{array}$ & $\begin{array}{r}1.17 \\
\pm 0.18\end{array}$ & $\begin{array}{r}1.03 \\
\pm 0.06\end{array}$ & $\begin{array}{c}0.94 \\
\pm 0.05\end{array}$ & $\begin{array}{c}1,01 \\
\pm 0.06\end{array}$ & $\begin{array}{l}1.04 \\
\pm 0.1\end{array}$ & $\begin{array}{c}0,94 \\
\pm 0.42\end{array}$ \\
\hline $\begin{array}{l}\text { Biomasses moyenne } \\
\text { individuelles }(\mathrm{mg})\end{array}$ & $\begin{array}{r}0.57 \\
\pm 0.04 \\
\end{array}$ & $\begin{array}{r}0.54 \\
\pm 0.04\end{array}$ & $\begin{array}{c}0.72 \\
\pm 0.23\end{array}$ & $\begin{array}{r}0.54 \\
+0.03\end{array}$ & $\begin{array}{c}0.67 \\
\pm 0,10\end{array}$ & $\begin{array}{l}0.51 \\
\pm 0.1\end{array}$ & $\begin{array}{r}0.49 \\
\pm 0.05 \\
\end{array}$ & $\begin{array}{c}0.54 \\
\pm 0.04 \\
\end{array}$ & $\begin{array}{r}0.56 \\
\pm 0.05\end{array}$ & $\begin{array}{l}0.66 \\
\pm 0.1\end{array}$ & $\begin{array}{c}1.11 \\
\pm 0.42\end{array}$ \\
\hline
\end{tabular}

La porosité apparente de la couche de surface influence de manière toujours significative les valeurs de densité et biomasse. Les substrats "fermés" (gros éléments dominants mais une forte proportion de sable, notés F) présentent des densités relatives et absolues plus faibles que les substrats “ouverts» (O). Une différence de même sens se retrouve pour les biomasses relatives, mais à l'inverse les biomasses absolues sont en moyenne plus élevées sur les substrats fermés. Ceci semble dû, comme pour les substrats sableux, à des individus plus gros, mais les différences de biomasse individuelle moyenne ne sont pas significatives.

La prise en compte simultanée des deux caractéristiques, diamètre et ouverture, aboutit à des différences très significatives $(p<0,01)$ pour les densités et biomasses relatives et absolues entre les deux groupes extrêmes ( $B-O, n=160$ et $G-F, n=35$ )

Effet vitesse - La vitesse influence de manière significative les densités brutes et relatives. La tendance générale des densités brutes suggère une augmentation avec la vitesse, mais une discontinuité très nette apparaît pour la gamme $60-70 \mathrm{~cm} / \mathrm{s}$ (V8) qui présente des valeurs significativement plus faibles que les classes qui l'encadrent (Tableau II). L'influence de la vitesse est encore plus nette sur les densités relatives (Figure 1). On retrouve la même tendance générale à l'augmentation, mais deux discontinuités significatives (Tableau III) apparaissent au niveau des classes V4 et V8 pour lesquelles les densités relatives sont plus faibles. 
Tableau II : Moyennes (t erreur standard) des densités et biomasses absolues et relatives, et des biomasses individuelles des invertébrés benthiques pour chaque classe de vitesses.

Table II : Means ( \pm standard error) of relative and absolute densities and biomass, and of individual biomass of benthic invertebrates in each velocity class.

\begin{tabular}{|c|c|c|c|c|c|c|c|c|c|c|c|c|}
\hline $\begin{array}{c}\text { (Classes de vitessers } \\
(\mathrm{cm} / \mathrm{s})\end{array}$ & $\begin{array}{l}v \mid \\
|0-5|\end{array}$ & $\begin{array}{c}V 2 \\
{[5-10]}\end{array}$ & $\begin{array}{c}\mathrm{V} 3 \\
{[10-20[}\end{array}$ & $\begin{array}{c}V 4 \\
|20-30|\end{array}$ & $\begin{array}{c}V 5 \\
{[30-40 \mid}\end{array}$ & $\begin{array}{c}\mathrm{V} 6 \\
{[40-50[}\end{array}$ & $\begin{array}{c}V 7 \\
{[50-60 \mid}\end{array}$ & $\begin{array}{c}V 8 \\
{[60-70]}\end{array}$ & $\begin{array}{c}\text { V9 } \\
{[70-80]}\end{array}$ & $\begin{array}{l}V 10 \\
{[80-90[}\end{array}$ & $\begin{array}{l}\text { V1] } \\
{[90-100]}\end{array}$ & $\begin{array}{c}V 12 \\
{[100-150[}\end{array}$ \\
\hline$n$ & 36 & 20 & 59 & 38 & 51 & 35 & $\overline{25}$ & 29 & 27 & 18 & 11 & 22 \\
\hline Densités & 5132.8 & 4908.8 & 5185.4 & 5473.7 & 7094.1 & 5882.3 & 6591.0 & 3951.4 & 7807.4 & 5646.7 & 8070.0 & 5254.3 \\
\hline ahsolues (ind./m2) & \pm 991.6 & \pm 555.8 & \pm 471.3 & \pm 488.4 & \pm 837.3 & $\pm 930,3$ & $\pm 696,0$ & \pm 524.1 & \pm 1674.0 & \pm 1237.7 & \pm 1799.5 & \pm 6.38 .2 \\
\hline Biomasses & 3747.5 & 3060.1 & 2726.5 & 2363.5 & 2891.3 & 2287,9 & 2746.1 & 2080.7 & 3.365 .4 & 2284.8 & 4046.4 & .3164 .7 \\
\hline absolues (mg/m2) & \pm 815.5 & \pm 9.39 .6 & \pm 407.4 & \pm 368.8 & \pm 306.9 & \pm 315.3 & \pm 384.7 & \pm 30.7 .7 & \pm 502.6 & \pm 423.8 & \pm 994.4 & \pm 572.4 \\
\hline $\begin{array}{l}\text { Biomasse } \\
\text { individuefles (mg) }\end{array}$ & $\begin{array}{c}0.91 \\
\pm 0.22\end{array}$ & $\begin{array}{r}0.64 \\
\pm 0.18\end{array}$ & $\begin{array}{c}0.57 \\
+0.07\end{array}$ & $\begin{array}{c}0.44 \\
\pm 0.05\end{array}$ & $\begin{array}{r}0.53 \\
\pm 0.07\end{array}$ & $\begin{array}{c}0.47 \\
\pm 0.06\end{array}$ & $\begin{array}{r}0,46 \\
\pm 0.06\end{array}$ & $\begin{array}{c}0.62 \\
\pm 0.1(0)\end{array}$ & $\begin{array}{c}0.52 \\
\pm 0,05\end{array}$ & $\begin{array}{r}0.56 \\
\pm 0.09\end{array}$ & $\begin{array}{r}0.46 \\
+0.07\end{array}$ & $\begin{array}{c}0.62 \\
+0,07\end{array}$ \\
\hline
\end{tabular}

Tableau III : Visualisation des tests de Mann-Whitney de comparaison des moyennes de densité relative par classe de vitesse (classes 1 à 12, demi-matrice du haut), et par groupe de classes de vitesses (groupes A à E, demi-matrice du bas).

Table III : Mann-Whitney tests of mean relative densities per velocity class (classes 1 to 12, upper half matrix) and per group of velocity classes (groups A to E, lower half matrix).

\begin{tabular}{|c|c|c|c|c|c|c|c|c|c|c|c|c|c|c|}
\hline \multirow{2}{*}{$\begin{array}{l}\text { Vitesse } \\
\mathrm{cm} / \mathrm{s}\end{array}$} & \multirow{2}{*}{\multicolumn{2}{|c|}{$\begin{array}{l}\text { Classe } \\
\text { de vitesse }\end{array}$}} & 1 & 2 & 3 & \multirow{2}{*}{$\frac{4}{B}$} & 5 & 6 & 7 & \multirow{2}{*}{$\frac{8}{D}$} & 9 & 10 & 11 & 12 \\
\hline & & & \multicolumn{3}{|c|}{$\mathrm{A}$} & & \multicolumn{3}{|c|}{$\mathrm{C}$} & & \multicolumn{4}{|c|}{$\mathrm{E}$} \\
\hline \multirow{3}{*}{$\begin{array}{l}{[0-5[} \\
{[5-10[} \\
{[10-20[}\end{array}$} & 1 & \multirow{3}{*}{ A } & \multirow[t]{3}{*}{1} & - & - & - & - & - & - & - & - & - & - & - \\
\hline & 2 & & & \multirow[t]{2}{*}{ I } & - & - & - & - & - & - & - & $*$ & - & - \\
\hline & 3 & & & & 1 & - & - & - & - & - & - & $*$ & - & - \\
\hline$[20-30]$ & 4 & B & \multicolumn{3}{|c|}{-} & 1 & $m$ & - & $m$ & - & $m$ & $*$ & 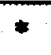 & - \\
\hline$[30-40[$ & 5 & \multirow{3}{*}{$\mathrm{C}$} & \multirow{3}{*}{\multicolumn{3}{|c|}{-}} & \multirow{3}{*}{$* *$} & \multirow[t]{3}{*}{1} & - & - & $*$ & - & - & - & - \\
\hline$[40-50[$ & 6 & & & & & & & \multirow[t]{2}{*}{1} & - & + & - & - & - & - \\
\hline$[50-60[$ & 7 & & & & & & & & 1 & $*$ & - & - & - & - \\
\hline$[60-70]$ & 8 & D & \multicolumn{3}{|c|}{ - } & & \multicolumn{3}{|c|}{$* *$} & 1 & $*$ & $* *$ & $\Rightarrow$ & - \\
\hline$[70-80[$ & 9 & \multirow{4}{*}{$\mathrm{E}$} & \multirow{4}{*}{\multicolumn{3}{|c|}{$* *$}} & \multirow{4}{*}{$* *$} & \multirow{4}{*}{\multicolumn{2}{|c|}{ - }} & & \multirow{4}{*}{$* * *$} & \multirow[t]{4}{*}{1} & - & - & - \\
\hline$[80-90[$ & 10 & & & & & & & & & & & \multirow[t]{3}{*}{1} & - & - \\
\hline$[90-100[$ & 11 & & & & & & & & & & & & \multirow[t]{2}{*}{1} & - \\
\hline$[100-150]$ & 12 & & & & & & & & & & & & & 1 \\
\hline
\end{tabular}

${ }^{\star \star}: p \leq 0,01 ;{ }^{\star}: p<0,05 ;-: p>0,05$. 


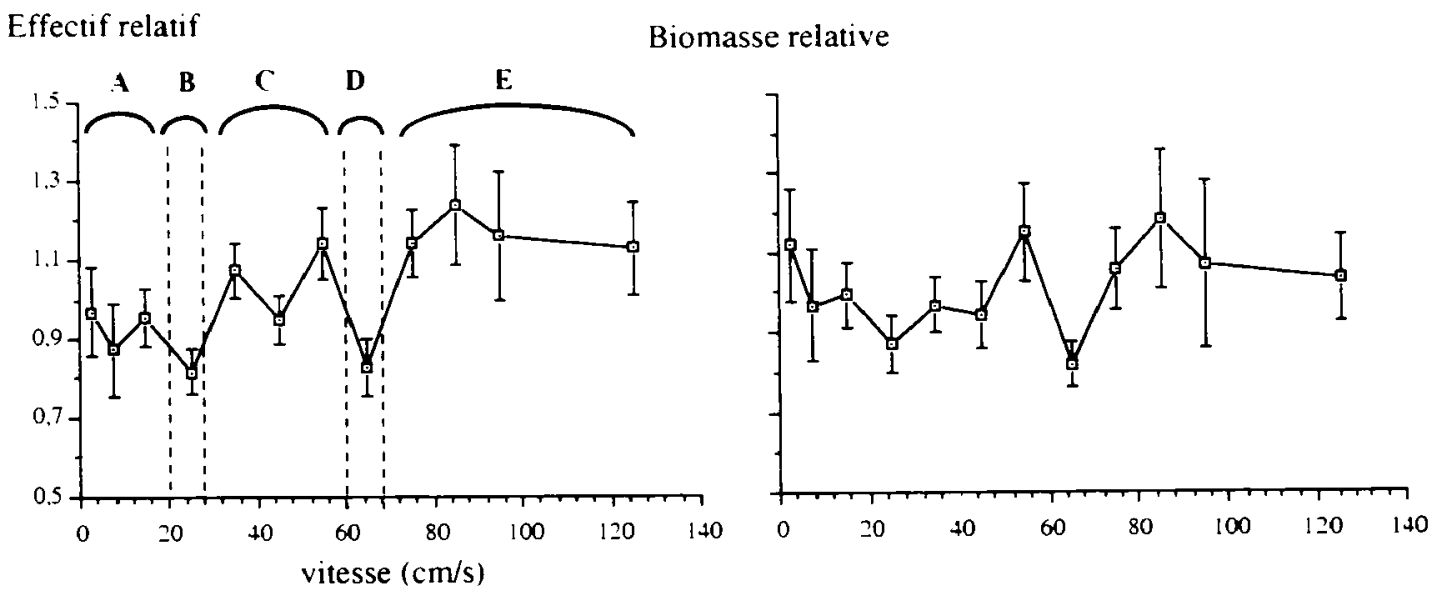

\section{Figure 1 : Moyennes ( \pm erreur standard) des densités et biomasses relatives par classe de vitesses.}

Figure 1 : Mean ( \pm standard error) relative densities and biomass in each velocity class.

Les profils de biomasses diffèrent des profils de densité par la présence de valeurs élevées à la fois pour de très fortes et très faibles vitesses. La biomasse moyenne des individus semble plus élevée dans les zones de faible courant. La courbe des biomasses relatives présente, comme pour les densités relatives, des minimum au niveau des classes V4 et V8, avec une discontinuité marquée pour V8. Mais l'influence de la vitesse sur les valeurs de biomasse n'est jamais statistiquement significative.

Une seconde analyse a été réalisée en regroupant les classes consécutives qui ne présentent pas de différences significatives en densités relatives (Tableau III). L'influence du facteur vitesse est alors hautement significative $(p=0,0001)$. Les densités relatives sont plus basses dans les vitesses faibles $(A: 0-20 \mathrm{~cm} / \mathrm{s})$ que dans les vitesses forte $(E: 70-150 \mathrm{~cm} / \mathrm{s})$, mais la gamme intermédiaire ( $\mathrm{C}: 30-60 \mathrm{~cm} / \mathrm{s}$ ) ne diffère pas significativement des deux extrêmes. Entre ces trois classes s'observent deux discontinuités. Les densités relatives sont plus faibles dans la gamme $B(20-30 \mathrm{~cm} / \mathrm{s})$ que dans la gamme $C(p<0,01)$, et la plage $D$ $(60-70 \mathrm{~cm} / \mathrm{s})$ diffère significativement de celles qui l'encadrent $(C-E)$. Ce phénomène n'est pas dû à un biais de l'échantillonnage, car il n'y a aucune prédominance particulière d'une rivière ou d'un type de substrat dans cette classe de vitesse.

Le profil de variation des densités brutes est relativement similaire, sauf pour la discontinuité de la plage $B$. Les vitesses faibles présentent des densités plus basses que les vitesses intermédiaires $(p<0,05)$, et la discontinuité de la plage $D$ est toujours significative $(p=0,01)$.

Le même découpage en 5 classes pour les biomasses brutes et relatives aboutit à une distribution bimodale. Les valeurs élevées se retrouvent à la fois dans les classes de vitesses faibles et fortes, et les deux discontinuités restent marquées. Les biomasses individuelles sont sensiblement plus élevées dans les vitesses faibles. Cependant, ces variations ne sont pas statistiquement significatives.

Effet hauteur - La hauteur d'eau influence significativement les densités brutes et relatives, mais les deux profils sont assez différents. Les minima de densité brute s'observent dans les hauteurs d'eau faibles $(\mathrm{H} 1)$ et fortes $(\mathrm{H} 6)$, et le maximum dans la classe H2 (Tableau I). En densités relatives, le maximum correspond aux plus faibles profondeurs $(\mathrm{H} 1)$, mais la variabilité est très forte dans cette classe. La classe $\mathrm{H} 3$ présente des valeurs plus faibles que la classe $\mathrm{H} 2(\mathrm{p}<0,05)$, et la diminution des densités au-delà de $50 \mathrm{~cm}$ est également significative. 
Pour les biomasses, les profils de valeurs brutes et relatives sont inversés. Les fortes biomasses absolues apparaissent dans les plus grandes profondeurs ( $>40 \mathrm{~cm}$ ), alors que la biomasse relative semble plus élevée dans les faibles profondeurs $(\mathrm{H} 1)$. Le profil des biomasses individuelles suggère des organismes sensiblement plus gros au delà de $50 \mathrm{~cm}$ de profondeur, mais aucune de ces variations n'apparaît statistiquement significative.

Influence relative des trois variables - Les variations maximales des variables biologiques (densités et biomasses) dues à chacun des trois paramètres physiques (hauteur, vitesse et substrat), ont été estimées par les différences entre les valeurs des classes présentant les minima et maxima de densité et biomasses, exprimées en \% de la moyenne de l'ensemble des prélèvements. Les écarts sont généralement plus forts sur les données brutes que sur les valeurs relatives (tableau IV). L'effet substrat (diamètre et ouverture) est de l'ordre de $28 \%$ et $18 \%$ respectivement sur les densités brutes et relatives. L'effet vitesse est de l'ordre de $70 \%$ pour les densités et biomasses brutes, et de $40 \%$ en valeurs relatives. L'effet hauteur est de l'ordre de $45 \%$ sur les densités.

Tableau IV : Ecart maximal observé sur chaque variable biologique (exprimé en \% de la moyenne de l'ensemble des prélèvements) pour chaque paramètre physique, et pour les combinaisons d'habitat les plus favorables et défavorables.

Table IV : Maximal observed variation for each biological variable (expressed as \% of the mean of all samples) due to each physical parameter alone, and between the most and the less favourable habitat combinations.

\begin{tabular}{|l|c|c|c|c|}
\cline { 2 - 5 } \multicolumn{1}{c|}{} & \multicolumn{3}{c|}{ Ecart maximal observé } \\
\cline { 2 - 5 } \multicolumn{1}{c|}{} & $\begin{array}{c}\text { Densités } \\
\text { absolues }\end{array}$ & $\begin{array}{c}\text { Densités } \\
\text { relatives }\end{array}$ & $\begin{array}{c}\text { Biomasses } \\
\text { absolues }\end{array}$ & $\begin{array}{c}\text { Biomasses } \\
\text { relatives }\end{array}$ \\
\hline Ouverture substrat (O/F) & $27 \%$ & $17 \%$ & $-8 \%$ & $14 \%$ \\
Taille substrat (B/G) & $29 \%$ & $19 \%$ & $40 \%$ & $17 \%$ \\
Substrat global (B-O/G-F) & $53 \%$ & $30 \%$ & $32 \%$ & $31 \%$ \\
Vitesse & $71 \%$ & $42 \%$ & $70 \%$ & $36 \%$ \\
Hauteur & $48 \%$ & $43 \%$ & $47 \%$ & $23 \%$ \\
Combinaison Sub. Vit. & $65 \%$ & $88 \%$ & $36 \%$ & $43 \%$ \\
(B-O-V10/G-V4 ou V8) & & & & \\
\hline
\end{tabular}

En sélectionnant les prélèvements qui appartiennent comme précédemment aux classes présentant les valeurs maximales et minimales des variables biologiques, il est possible d'estimer l'amplitude de la variation due à la combinaison de plusieurs paramètres physiques. Pour le seul substrat, l'écart entre les combinaisons B-O et G-F est de $53 \%$ sur les densités absolues, et de l'ordre de $30 \%$ sur les densités relatives et les biomasses. En croisant le substrat et la vitesse, les valeurs maximales (B-O-V10) et minimales (G-V4 ou V8) correspondantes sont respectivement de 1,62 et 0,74 pour la densité relative, soit une variation de $88 \%$, et de 1,23 et 0,80 pour la biomasse relative, soit $43 \%$. Pour les mêmes combinaisons d'habitat, les variations absolues sont inférieures aux variations relatives. En rajoutant l'effet du substrat fermé à la combinaison la plus défavorable, les valeurs de densité et biomasse relative tomberaient respectivement à 0,18 et 0,32 , mais ces chiffres correspondant à seulement 3 prélèvements ne sont pas significatifs. 


\section{DISCUSSION}

Les invertébrés répondent quantitativement aux variations de leur habitat physique, mais aussi aux caractéristiques physico-chimiques de l'eau, et à la disponibilité des ressources trophiques. Or, ces derniers facteurs peuvent varier à l'échelle d'une région écologique (température, dureté, $\mathrm{pH}$, turbidité), ou d'un tronçon de cours d'eau, en fonction de la morphologie et de l'environnement végétal (périphyton, matière organique). Les cycles saisonniers, dépendant d'un régime hydrologique régional, induisent aussi de fortes variations d'effectifs. Tous ces facteurs influencent les valeurs brutes de densité et de biomasse, et les profils correspondant aux paramètres physiques peuvent s'en trouver biaisés dans la mesure où l'échantillonnage n'est pas stratifié par région et par saison. En revanche, les valeurs relatives par échantillon isolent une variation due uniquement à l'habitat aquatique, la répartition dans les principaux habitats disponibles des invertébrés présents dans une station à une date donnée. Ces valeurs relatives expriment donc une "habitabilité" des conditions physiques. Cependant, la réponse aux variations de l'habitat dues aux différences inter-sites (fonction de la nature régionale des matériaux du substrat et de la pente) ou inter-saisons (fonction du débit) n'est pas intégrée dans la variation des densités et biomasses relatives à chaque échantillon. Ceci explique que les variations brutes pour chaque paramètre soient généralement supérieures à celles des valeurs relatives, mais il reste à faire la part de ce qui est dû uniquement à l'habitat physique. A noter que la variation relative est supérieure à la variation brute lorsque l'on combine les variables vitesse-substrat, ce qui renforce la signification de "préférence d'habitat" des valeurs relatives.

Si l'on tient compte à la fois de l'amplitude et de la significativité des variations biologiques induites, le substrat apparaît comme un facteur très important puisqu'il influence significativement les densités et les biomasses. Les paramètres hydrauliques n'ont une influence significative que sur les densités, même si l'on réduit le nombre de classes. L'effet vitesse est sensiblement supérieur à l'effet hauteur.

Les résultats ci-dessus permettent de quantifier l'effet global de phénomènes déjà connus, comme le caractère attractif des substrats de gros diamètre, ou l'effet limitant d'une fermeture des substrats grossiers par les sables (MINSHALL, 1984). Ce dernier point corrobore les observations relatives à l'influence déterminante de la porosité, inversement liée au pourcentage des fractions fines, sur la colonisation par les invertébrés des couches profondes du sédiment (MARIDET et al., 1992). En revanche, la très forte hétérogénéité des peuplements sur les substrats sableux n'a pas été souvent observée. Selon les courbes de préférence existantes (WATERS, 1976 ; MILHOUS, 1990), les sables sont considérés comme des substrats abritant de très faibles densités d'invertébrés. Ce point doit être fortement nuancé, car les dépôts sableux peuvent abriter une faune aussi abondante que les substrats pierreux. Ceci dépend probablement de la quantité de débris végétaux, généralement colonisés par de gros déchiqueteurs, accumulés sur ces substrats, ce qui explique probablement les plus fortes biomasses brutes observées sur les substrats fermés. Mais les biomasses relatives montrent que les substrats ouverts sont globalement "préférés" par la faune. La simple mention de l'élément granulométrique dominant ou médian ne suffit donc pas pour évaluer l'habitabilité d'un substrat ; il faut prendre en compte également les fractions fines, et probablement la stabilité et les débris végétaux pour les substrats sableux.

Pour la vitesse, nos résultats diffèrent nettement des courbes de préférence existantes. Premièrement, l'effet des faibles vitesses est loin d'être aussi limitant que le suggère JOWETT (1992, d'après WATERS, 1976) ; la courbe utilisée par MILHOUS (1990) est nettement plus proche de nos valeurs. Deuxièmement, nous n'observons pas, contrairement à ces auteurs, une diminution brutale des peuplements dans la gamme $100-150 \mathrm{~cm} / \mathrm{s}$. Les densités et biomasses relatives ne diffèrent pas de la gamme de vitesse précédente, et la variabilité n'est pas plus forte. Nos données suggèrent trois gammes de vitesses d'habitabilité croissante, $0-20,30-60$, et $70-140 \mathrm{~cm} / \mathrm{s}$. Ces résultats rappellent les gammes de vitesses proposées par DUMONT et RIVIER (1981) d'après la composition qualitative des peuplements. Enfin, et c'est le point le plus surprenant, les minima de densité et biomasse apparaissent entre ces classes, au niveau de deux discontinuités qui semblent évitées de manière significative par les invertébrés. 
De telles discontinuités n'avaient jamais été signalées, et ce résultat demanderait à être confirmé sur d'autres données. Certaines hypothèses explicatives peuvent toutefois être avancées. D'une part, la vitesse de $60 \mathrm{~cm} / \mathrm{s}$ correspondrait à la limite supérieure pour le développement optimum du périphyton (HORNER et al., 1990) qui jouerait un rôle attractif majeur sur la faune. D'autre part, les microzones turbulentes (nombre de Froude $>1$ ) associées aux fortes vitesses sur les substrats de blocs pourraient constituer des conditions très favorables pour la faune (NEWBURY, STATZNER, comm. pers.). L'effet antagoniste de ces deux phénomènes pourrait expliquer la discontinuité de la gamme $60-70 \mathrm{~cm} / \mathrm{s}$.

L'influence de la hauteur d'eau est importante. Des différences notables apparaissent avec les courbes existantes qui considèrent les faibles profondeurs très limitantes. Nos résultats suggèrent, au contraire, une préférence des invertébrés pour les profondeurs $<20 \mathrm{~cm}$. Mais la baisse de densité au-delà de $50 \mathrm{~cm}$ pourrait être due en partie à une diminution de l'efficacité de l'échantillonnage au filet Surber.

En conclusion, les données ci-dessus constituent une première étape pour l'élaboration de courbes de préférence globales pour les invertébrés, intégrables dans des modèles de type microhabitat pour une assez large gamme de rivières salmonicoles. II sera préférable d'utiliser les densités relatives, dont les variations sont toujours significatives, et qui traduisent l'influence globale, minimale mais certaine, des paramètres morphodynamiques sur les invertébrés. L'utilisation conjointe des densités et biomasses permettrait aussi d'évaluer une productivité potentielle des différents habitats en tenant compte de la biomasse individuelle moyenne des organismes des principaux groupes faunistiques (MORIN et BOURASSA, 1992). Ceci conduirait à une meilleure estimation des zones de production de nourriture pour le poisson. En revanche, l'explication des distributions observées des invertébrés nécessitera une analyse à des niveaux taxonomiques et fonctionnels plus précis.

\section{REMERCIEMENTS}

Merci à François VANCAYSEELE, Gaït SUARD, Catherine AMBROISE-RENDU, Sylvie VALENTIN, ainsi qu'aux membres et nombreux stagiaires du laboratoire pour la collecte des données.

\section{BIBLIOGRAPHIE}

COLLIER K.J., 1993. Flow preferences of larval Chironomidae (Diptera) in Tongariro River, New Zealand. New Zealand J. Marine Freshwater Research, 27, 219-226.

COTTA-RAMUSINO M., CROSA G., BUFFAGNI A., 1991. Microhabitat preferences of benthic fauna (Ephemeroptera) in plane-springs (fontanili). Verh. Internat. Verein. Limnol., 24, 1626-1628.

DUMONT B., RIVIER B., 1981. La notion de débit réservé biologique. CEMAGREF QEPP Aix-en-Provence.

GORE J.A., JUDY R.D. Jr., 1981. Predictive models of benthic macroinvertebrate density for use in instream flow and regulated flow management. Can. J. Fish. Aquat. Sci., 38, 1363-1370.

HORNER R.R., WELCH E.B., SEELEY M.R., JACOBY J.M., 1990. Responses of periphyton to changes in current velocity, suspended sediment and phosphorus concentration. Freshwater Biol., 24, 215-232.

JOWETT I.G., 1992. Models of the abundance of large brown trout in New Zealand rivers. North Am. J. Fisheries Manag., 12, 417-432.

JOWETT I.G., RICHARDSON J., 1990. Microhabitat preferences of benthic invertebrates in a New Zealand river and the development of in-stream flow-habitat models for Deleatidium spp. New Zealand J. Marine Freshwater Research, 24, 19-30. 
JOWETT I.G., RICHARDSON J., BIGGS B.J.F., HICKEY C.W., QUINN J.M., 1991. Microhabitat preferences of benthic invertebrates and the development of generalised Deleatidium spp. habitat suitability curves, applied to four New Zealand rivers. New Zealand J. Marine Freshwater Research, 25, 187-199.

MARIDET L., WASSON J.G., PHILIPPE M., 1992. Vertical distribution of fauna in the bed sediment of three running water sites : influence of physical and trophic factors. Regulated Rivers : Research \& Management, 7, 45-55.

MILHOUS R.T., 1990. Instream habitat considering hydro-peaking. In : KHANBILVARDI R.M. \& GOOCH T.C. (eds), Optimizing the resources for water management, 593598, American Society of Civil Engineers, New York.

MINSHALL G.W., 1984. Aquatic insect-substratum relationships. In : RESH V.M. \& ROSENBERG D.M. (eds), Ecology of aquatic insects, 358-400.

MORIN A., HARPER P.P., PETERS R.H., 1986. Microhabitat-Preferences curves of blackfly larvae (Diptera : simuliidae) : a comparison of three estimation methods. Can. J. Fish. Aquat. Sci., 43, 1235-1241.

MORIN A., BOURASSA N., 1992. Modèles empiriques de la production annuelle et du rapport P/B d'invertébrés benthiques d'eau courante. Can. J. Fish. Aquat. Sci., 49, 532-539.

ORTH D.J., MAUGHAN O.E., 1983. Microhabitat preferences of benthic fauna in a woodland stream. Hydrobiologia, 106, 157-168.

STATZNER B., GORE J.A., RESH V.H., 1988. Hydraulic stream ecology : observed patterns and potential applications. J. N. Am. Benthol. Soc., 7, 307-360.

WASSON J.G., DUMONT B., TROCHERIE F., 1981. Protocole de description des habitats aquatiques et de prélèvement des invertébrés benthiques dans les cours d'eau. CEMAGREF Lyon, DQEPP, $18 \mathrm{p}$.

WATERS B.F., 1976. A methodology for evaluating the effects of different streamflows on salmonid habitat. In : ORSBORN J.F. \& ALLMAN C.H. (eds), Instream Flow Needs, 254-266, American Fisheries Society, Western Division, Bethesda, Maryland. 